\title{
Perfil nutricional de indivíduos com transtorno mental, usuários do Serviço Residencial Terapêutico, do município de Alfenas - MG
}

\author{
Priscila Cristina de Oliveira GARCIA ${ }^{1}$ \\ Juliana Cristina MOREIRA ${ }^{1}$ \\ Marcos Coelho BISSOLI ${ }^{2}$ \\ Tania Mara Rodrigues SIMÕES ${ }^{2 *}$
}

\author{
${ }^{1}$ Discente, Curso de Nutrição, Faculdade de Nutrição, Universidade Federal de Alfenas (UNIFAL-MG). \\ E-mail: priscila_cog@hotmail.com; julianac_moreira@hotmail.com. \\ ${ }^{2}$ Mestre, docente da Faculdade de Nutrição, UNIFAL-MG. E-mail: mbissoli@gmail.com, \\ tania.simoes@unifal-mg.edu.br, taniamararsimoes@gmail.com* \\ "Autor para correspondência: \\ Faculdade de Nutrição \\ Universidade Federal de Alfenas \\ Rua Gabriel Monteiro da Silva, 700 - Centro \\ 37130-000 - Alfenas - MG - Brasil
}

\section{RESUMO}

Esquizofrenia é um transtorno mental grave e complexo que afeta $1 \%$ da população mundial. Sua etiologia está longe de ser elucidada e seu tratamento envolve o uso de fármacos antipsicóticos, o qual pode contribuir para o ganho de peso. Pacientes com esquizofrenia têm maior risco de obesidade, complicações metabólicas e cardiovasculares, em comparação à população em geral. Este estudo teve como objetivo avaliar o estado nutricional de indivíduos com transtorno mental, usuários do Serviço Residencial Terapêutico de Alfenas MG. Foram coletados dados sociodemográficos, clínicos e antropométricos dos indivíduos (n=49), nas sete Residências Terapêuticas do município. Aferiram-se peso atual, estatura e circunferência da cintura (CC). Dentre os usuários, $29 \%$ eram do gênero feminino e $71 \%$, do masculino. A idade média foi de 50,10 $\pm 11,09$ anos. A média ponderal foi de 72,00 $\pm 15,85 \mathrm{~kg}$. O índice de massa corporal (IMC) médio foi de 27,20 \pm $5,81 \mathrm{~kg} / \mathrm{m}^{2}$. Pela classificação do IMC, $14 \%$ dos usuários apresentavam magreza, $25 \%$ eutrofia e $61 \%$ excesso de peso. Todas as mulheres apresentaram CC acima do recomendado e entre os homens, 59\% se encontravam na mesma situação, indicando presença de obesidade central em ambos os gêneros. As doenças crônicas não transmissíveis (DCNT) encontradas nos usuários foram hipertensão arterial (12\%), diabetes mellitus (23\%) e diabetes mellitus +dislipidemias (6\%). Apesar de não ter havido associação entre 
excesso de peso e as DCNT, faz-se necessário monitorar a situação nutricional e de saúde desses indivíduos para melhoria dos cuidados prestados, com promoção de atenção nutricional num contexto multiprofissional e interdisciplinar.

Palavras chave: Residências Terapêuticas. Transtornos mentais. Esquizofrenia. Estado nutricional. Doenças crônicas não transmissíveis.

\section{ABSTRACT}

Schizophrenia is a severe mental disorder and complex affecting $1 \%$ of the world population. Its etiology is far from being elucidated and its treatment involves the use of antipsychotic drugs and may contribute to weight gain. Patients with schizophrenia are at increased risk of obesity and metabolic and cardiovascular complications, compared to the general population. This study aimed to assess the nutritional status of individuals with mental illness, users Therapeutic Residential Service, Alfenas - MG. Sociodemographic data, clinical and anthropometric subjects $(n=49)$ in seven therapeutic residences in the city. We measured current weight, height and waist circumference (WC). Among users, 29\% were female and $71 \%$ male. The mean age was $50.10 \pm 11.09$ years. The average weight was $72.00 \pm 15.85 \mathrm{~kg}$. The mean body mass index (BMI) was $27.20 \pm 5.81 \mathrm{~kg} / \mathrm{m}^{2}$. By BMI classification, $14 \%$ of users had thinness, $25 \%$ normal weight and $61 \%$ overweight. All women presented above recommended CC and 59\% among men $(n=19)$ were in the same situation, indicating the presence of central obesity in both genders. The chronic noncommunicable diseases (NCDs) found in users were hypertension (12\%), diabetes mellitus $(23 \%)+$ dyslipidemia and diabetes mellitus $(6 \%)$. Although there was no association between overweight and NCD, it is necessary to monitor the nutritional status and health of these individuals to improve of care, to promote nutritional care in a multidisciplinary and interdisciplinary context.

Keywords: Therapeutic homes. Mental disorders. Schizophrenia. Nutritional status. Chronic noncommunicable diseases.

\section{INTRODUÇÃO}

A reforma psiquiátrica brasileira, voltando-se para a atenção psicossocial em saúde mental, criou serviços substitutivos aos hospitais psiquiátricos, centrando-se no princípio da desinstitucionalização e reabilitação psicossocial (BRASIL, 2005). Nesse contexto, foram criados pelo Ministério da Saúde, os Centros de Atenção Psicossocial (CAPS), as Residências Terapêuticas, entre outros (MARTINS et al., 2012).

A esquizofrenia é um transtorno mental grave, que afeta $1 \%$ da população 
mundial (GAMA et al.,2004; RAHMOUNE et al., 2012).

O tratamento da esquizofrenia envolve o uso de fármacos antipsicóticos, o que tem sido de fundamental importância para reduzir o número e o tempo de internações hospitalares, possibilitando a permanência desses indivíduos junto a seus familiares, melhorando também sua aceitação pela comunidade (LEITÃO-AZEVEDO et al., 2006). Entretanto, o uso de alguns psicotrópicos pode contribuir para o ganho de peso nesse grupo de pacientes e que a obesidade nesses indivíduos pode levar à perda da autoestima, ao isolamento social e à estigmatização (ATTUX et al., 2009).

Zugno et al. (2012) relatam que a obesidade pode, ainda, levar a outras doenças crônicas não transmissíveis, como diabetes mellitus (DM), dislipidemias e outras alterações relacionadas à síndrome metabólica, aumentando o risco de desenvolvimento de doenças cardiovasculares, que são a maior causa de morte em indivíduos com esquizofrenia. Em pacientes com esquizofrenia, sedentarismo, dieta desbalanceada e uso de antipsicóticos são condições comumente presentes e contribuem para ganho ponderal (ATTUX et al., 2009).

Para prevenção e controle do ganho de peso e dos elevados riscos relatados nessa população, recomenda-se o acompanhamento com manutenção do cuidado ativo desses indivíduos, com orientação nutricional, de estilo de vida e nível de atividade (REIS et al., 2007; LEITÃO-AZEVEDO et al., 2007).

O presente estudo teve como objetivo avaliar o estado nutricional de indivíduos com transtorno mental, usuários do Serviço Residencial Terapêutico (SRT), do município de Alfenas - MG, etapa esta fundamental para nortear o tratamento nutricional e mudanças na qualidade de vida e de saúde dessa população.

\section{METODOLOGIA}

O presente trabalho foi desenvolvido como parte de um projeto de extensão, intitulado "De perto ninguém é normal: educação em nutrição e saúde para pessoas com transtorno mental".

Este estudo foi do tipo transversal, realizado no ano de 2012, nas sete residências do SRT, do município de Alfenas - MG, após aprovação pelo Comitê de Ética em Pesquisa (CEP) da UNIFAL-MG, processo $\mathrm{n}^{\circ} \mathrm{CAEE}$ 01426012.3.0000.5142. Todos os participantes e seus responsáveis assinaram o termo de consentimento livre e esclarecido (estruturado conforme Resolução 196/1996 do Conselho Nacional de Saúde - CNS), antes do início do estudo. 
A amostragem foi não probabilística, tendo sido considerado como universo amostral, todos os usuários cadastrados no SRT no período do estudo ( $\mathrm{n}=49$ ), de ambos os gêneros, maiores de 18 anos. No entanto, excluíram-se da avaliação antropométrica aqueles indivíduos que não quiseram ou não estavam em condições de participar $(n=5)$, contudo foram obtidos os dados clínicos e sociodemográfico de todos os usuários participantes do estudo.

Foi utilizado um instrumento para coleta de dados, aplicado com objetivo de avaliar as características sociodemográficas e clínicas da população estudada, tendo sido realizada com os responsáveis técnicos pelos usuários, chamados de referências técnicas das Residências Terapêuticas. As variáveis sociodemográficas utilizadas para o presente estudo foram idade, sexo e densidade domiciliar (número de moradores por cômodo). As variáveis clínicas foram diagnóstico clínico, presença ou não de doenças crônicas não transmissíveis e existência ou não de acompanhamento nutricional.

Os dados antropométricos foram obtidos nas Residências Terapêuticas, aferindo-se peso atual, altura e circunferência da cintura (CC) dos participantes, conforme preconizado pelo Sistema de Vigilância Alimentar e Nutricional - SISVAN, do
Ministério da Saúde (BRASIL, 2008), para os grupos etários estudados.

O estado nutricional dos indivíduos foi classificado pelo índice de massa corporal (IMC), conforme SISVAN (BRASIL, 2008), utilizando-se os critérios de Lipschitz e Organização Mundial da Saúde (OMS) para idosos e adultos, respectivamente. Foram utilizados, para os adultos, pontos de corte adaptando-se da seguinte forma: magreza $\left(<18,5 \mathrm{~kg} / \mathrm{m}^{2}\right)$, eutrofia $\left(18,5\right.$ a $\left.24,9 \mathrm{~kg} / \mathrm{m}^{2}\right)$, excesso de peso $\left(\geq 25 \mathrm{~kg} / \mathrm{m}^{2}\right)$. A CC foi classificada pelos pontos de corte adotados pelo SISVAN (BRASIL, 2008).

Em relação aos medicamentos utilizados por essa população, os antipsicóticos relatados foram divididos em três categorias, típicos, atípicos e clozapina, estudando-se seus efeitos colaterais em relação ao estado nutricional.

Para análise estatística, foi utilizado o programa EpiInfo 3.5.2. Os dados coletados foram apresentados de forma descritiva, com média e desvio-padrão, erro padrão, mediana e intervalo de $95 \%$ de confiança (IC 95\%). As associações entre dados paramétricos foram avaliadas por meio de análise de variância (ANOVA) e os dados não paramétricos, por meio do Teste U de Mann-Whitney. Todas as probabilidades de significância $\quad(p)$ apresentadas foram consideradas estatisticamente significativas quando os 
valores de $\quad \mathrm{p}<0,05 \quad$ (JEKEL; KATZ;

ELMORE, 2005).

\section{RESULTADOS E DISCUSSÃO}

Todos os usuários do SRT, do município de Alfenas, no período de realização deste estudo, apresentaram diagnóstico psiquiátrico de esquizofrenia residual. A caracterização da amostra está apresentada na Tabela 1. A idade média dos indivíduos foi de 50,10 $\pm 11,09$ anos, sendo $71 \%$ do sexo masculino e $29 \%$ do sexo feminino. A média de peso encontrada foi de $72,00 \pm 15,85 \mathrm{~kg}$. O IMC médio foi de 27,20 $\pm 5,81 \mathrm{~kg} / \mathrm{m}^{2}$, caracterizando excesso de peso. Segundo a classificação do IMC, dentre os adultos com excesso de peso $(n=22), 50 \%$ $(n=11)$ apresentavam sobrepeso, 32\% $(n=7)$ obesidade grau $\mathrm{I}, 14 \%(\mathrm{n}=3)$ obesidade grau II e 4\% ( $n=1)$ obesidade grau III. Avaliando todos os indivíduos, independente do grupo etário $\quad(n=44), 14 \% \quad(n=6) \quad$ apresentavam magreza, $25 \%(n=11)$ eutrofia e $61 \%(n=27)$, excesso de peso (Figura 1). Todas as mulheres apresentaram CC acima do recomendado e entre os homens, 59\% $(n=19)$ se encontravam na mesma situação, indicando presença de obesidade central em ambos os gêneros. Em estudo realizado por Vargas (2011), com população do gênero masculino, foi demonstrado que $50 \%$ apresentavam CC aumentada. Straker et al. (2005) encontraram que $92 \%$ da população com esquizofrenia, independente do gênero, apresentavam esta

Tabela 1 - Caracterização da população estudada, Alfenas-MG, 2012.

\begin{tabular}{ll}
\hline Características & Valor \\
\hline Idade (anos; média \pm DP) & $50,10 \pm 11,09$ \\
Adultos & $75,51 \%$ \\
Idosos & $24,49 \%$
\end{tabular}

Gênero

$\begin{array}{rr}\text { Masculino } & 71 \% \\ \text { Feminino } & 29 \%\end{array}$




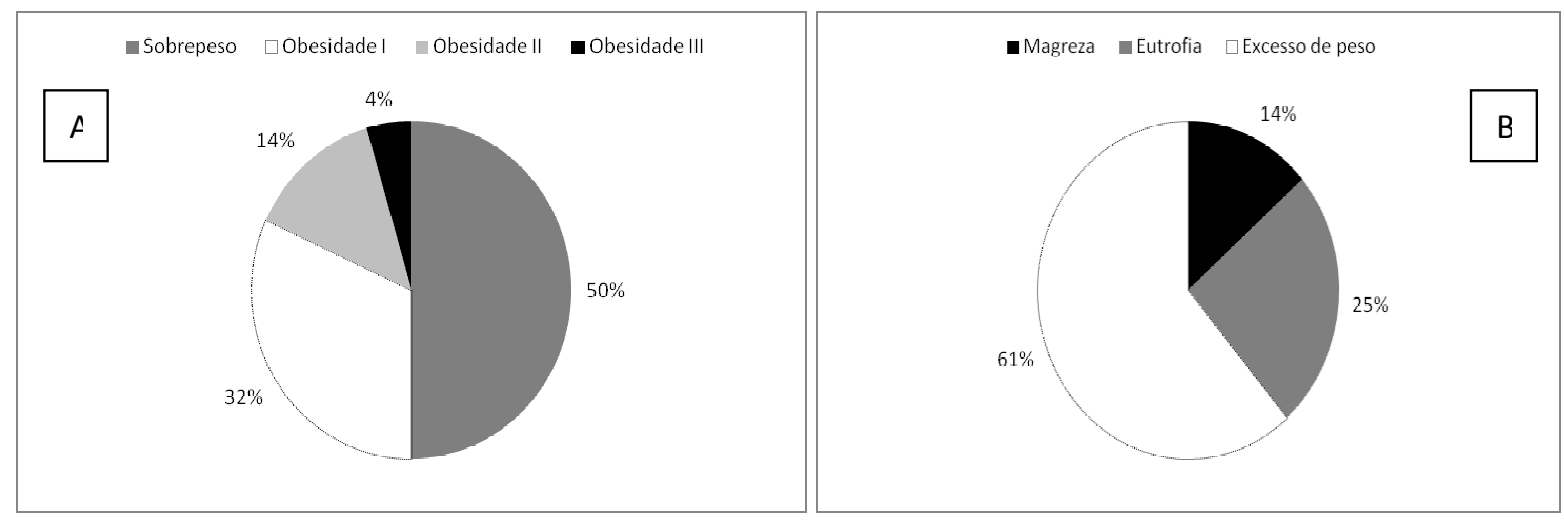

Figura 1 - Classificação de IMC dos adultos com excesso de peso (A) e de todos os usuários avaliados antropometricamente independente do grupo etário (B). Alfenas-MG, 2012.

medida antropométrica acima dos níveis de normalidade.

Costa (2009) relatou que a obesidade tornou-se mais comum entre indivíduos com esquizofrenia, quando comparado à população em geral, por causas multifatoriais e não bem definidas, sendo citadas a medicação antipsicótica, estilo de vida, maus hábitos alimentares e inatividade física. Outro estudo também mostrou resultados semelhantes (ZORTÉA et al., 2010) onde o sobrepeso e a obesidade foram prevalentes na população esquizofrênica, quando comparada com pacientes psiquiátricos sem o diagnóstico de esquizofrenia e a população em geral. $\mathrm{Na}$ população esquizofrênica foram encontrados $45 \%$ dos pacientes eutróficos, $30 \%$ com sobrepeso e $25 \%$ com obesidade. Também Leitão-Azevedo e colaboradores (2007), mostraram que a maioria de pacientes com esquizofrenia (70\%) apresentavam sobrepeso/obesidade quando comparados com a população geral $(30 \%)$.

Dados da Pesquisa de Orçamentos Familiares (POF) 2008-2009, no Brasil, indicaram um aumento de excesso de peso e obesidade na população com mais de 20 anos de idade, ao longo de 35 anos. O excesso de peso quase triplicou entre homens, sendo 50,1\% em 2008-09. Considerando as mulheres, $48 \%$ apresentavam excesso de peso. Com relação à obesidade, $12,4 \%$ dos homens e $16,9 \%$ das mulheres encontravamse nesse quadro nutricional (MELO, S/D).

A Figura 2 apresenta a frequência das doenças crônicas não transmissíveis (DCNT) na população estudada, sendo hipertensão arterial sistêmica (HAS; $\mathrm{n}=6)$, diabetes mellitus e dislipidemia (DM+dislipidemia; $\mathrm{n}=3$ ) e dislipidemias $(\mathrm{n}=11)$, sendo que 29 indivíduos não apresentaram nenhuma DCNT.

Alguns estudos anteriores mostraram uma distribuição diferente de DCNT entre 
pessoas com esquizofrenia. Vargas (2011), avaliando a presença de hiperglicemia em pacientes esquizofrênicos, encontrou uma prevalência de $30 \%$ desta alteração glicêmica, enquanto Leitão-Azevedo, 2006, encontraram prevalência de 40,34\% de hiperglicemia e $84,7 \%$ de dislipidemia em esquizofrênicos.

Em relação às DCNT relatadas, hipertensão arterial, diabetes mellitus e dislipidemias não se relacionaram com o estado nutricional de excesso de peso (Tabela 2). O IMC não teve significância estatística quando relacionado com a existência ou não de acompanhamento nutricional $(\mathrm{p}=0,1172)$, presença de hipertensão arterial $(\mathrm{p}=0,3528)$, diabetes mellitus $(\mathrm{p}=0,6309)$ e dislipidemia $(\mathrm{p}=0,5081)$, como mostrado na Tabela 3 .
Ressalta-se que, nenhum usuário apresentou acompanhamento nutricional realizado por nutricionista. Quando existente, o acompanhamento era realizado por médico. O acompanhamento nutricional feito pelo profissional nutricionista é de grande importância, como discutido nos estudos de Kalarchian et al. (2005) e Aquila e Emanuel (2000) apud Attux et al. (2009). No primeiro, foi realizada intervenção conduzida por psicóloga e nutricionista e observada perda de peso estatisticamente significativa; no segundo, a redução não teve significância estatística, mas, ainda assim, os autores concluíram que é relevante e há necessidade de intervenções nutricionais para prevenção e

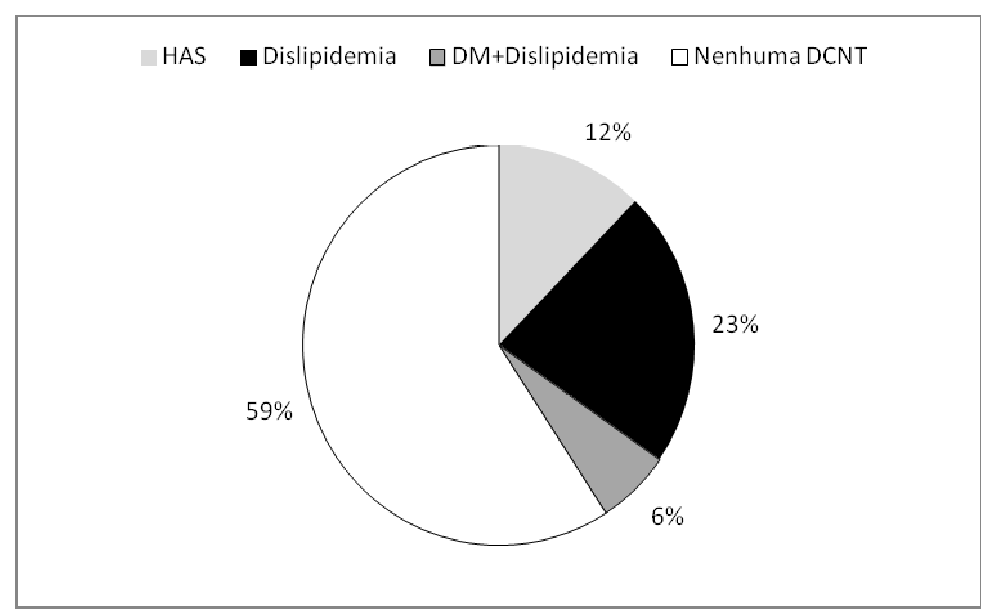

Figura 2 - Distribuição (\%) de DCNT nos indivíduos avaliados. Alfenas-MG, 2012. 
Tabela 2 - Associação do excesso de peso com as DCNT, nos usuários do SRT, Alfenas-MG, 2012; valores de razão de chance e IC $95 \%$.

\begin{tabular}{llll}
\hline Exposição & Desfecho & Razão de chance & IC 95\% \\
\hline \multirow{3}{*}{ Excesso de peso } & HAS & 1,74 & $0,29-10,52$ \\
& DM & 0,38 & $0,03-4,55$ \\
& Dislipidemias & 1,70 & $0,47-6,11$ \\
\hline
\end{tabular}

HAS: hipertensão arterial sistêmica, DM: diabetes mellitus.

Tabela 3 - Relação do estado nutricional com DCNT e acompanhamento nutricional, nos usuários do SRT, AlfenasMG, 2012; média de IMC e valores de p.

\begin{tabular}{lll}
\hline Variável & Média de IMC $\left(\mathrm{em} \mathrm{Kg} / \mathrm{m}^{2}\right)$ & $\mathrm{p}$ (Teste ANOVA) \\
\hline Acompanhamento Nutricional & & \\
$\quad$ Sim & 26,45 & 0,1172 \\
$\quad$ Não & 29,73 & \\
Hipertensão & \\
$\quad$ Sim & 29,27 & 0,3528 \\
Não & 26,87 & \\
Diabetes & & \\
Sim & 25,62 & 0,6309 \\
Não & 27,31 & \\
Dislipidemia & \\
$\quad$ Sim & 28,10 & 0,5081 \\
Não & 26,81 & \\
\hline
\end{tabular}

tratamento do ganho de peso associado ao uso de antipsicóticos.

Mesmo que não tenha sido verificada associação entre estado nutricional e DCNT, vale frisar que excesso de peso é fator de risco tanto para doenças cardiovasculares quanto para o desenvolvimento de síndrome metabólica (SM). De acordo com a I Diretriz Brasileira de Diagnóstico e Tratamento da Síndrome Metabólica (SOCIEDADE BRASILEIRA DE CARDIOLOGIA, 2005), esta é caracterizada pela presença de três ou mais dos seguintes fatores: obesidade central, hipertrigliceridemia, baixos níveis de colesterol ligado à proteína de alta densidade (HDL-colesterol), hipertensão arterial e hiperglicemia de jejum, necessitando de tratamento multiprofissional e interdisciplinar em saúde. Em revisão bibliográfica realizada por Teixeira (2007), foi visto que pacientes com transtornos mentais mais graves, como esquizofrenia e transtornos esquizoafetivos, apresentam probabilidade maior de serem acometidos por SM.

Em relação à variável densidade domiciliar, nos domicílios com maior número de morador por cômodo, foram encontrados os quadros de magreza, eutrofia e excesso de peso, sendo o último o de maior prevalência (Figura 3), enquanto que nas residências com menor densidade domiciliar, foi diagnosticado apenas excesso de peso. Foi mostrado, 
portanto, que a densidade domiciliar interfere no estado nutricional $(\mathrm{p}=0,0402)$, situação também evidenciada por Rissin (2006) em estudo com crianças do estado brasileiro de Pernambuco, onde o número de moradores por cômodo se relacionou com o estado nutricional de baixo peso. Baseando-se nesses dados, arriscamo-nos a inferir que, tanto crianças quanto indivíduos com transtorno mental grave dependem de cuidado ativo em relação à alimentação e saúde, e que a densidade domiciliar pode interferir na qualidade da atenção prestada por seus cuidadores.

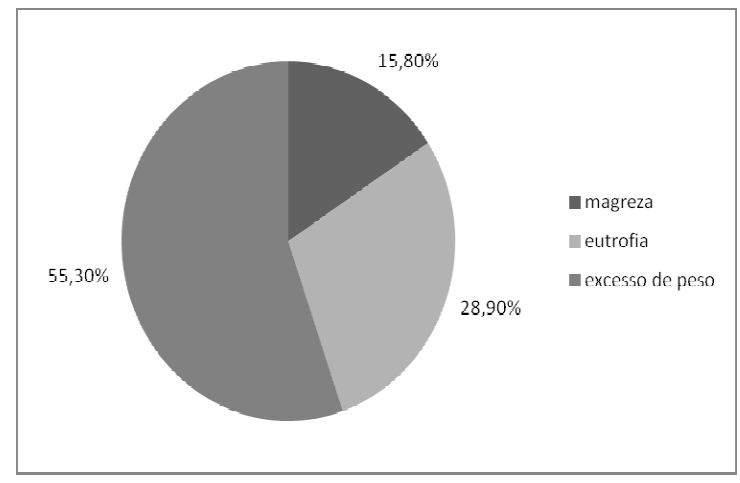

Figura 3 - Estado nutricional dos indivíduos moradores das Residências Terapêuticas com maior densidade domiciliar, Alfenas-MG, 2012.

Quanto às drogas antipsicóticas, estas contribuíram para a evolução do tratamento da esquizofrenia, prevenindo e tratando os sintomas, além de favorecer a reinserção social de esquizofrênicos (ATTUX et al., 2009). Entretanto, estudos evidenciaram que pacientes em tratamento com antipsicóticos apresentam importante ganho de peso (LEITÃO-AZEVEDO, 2006).
Algumas explicações para o ganho de peso causado por antipsicóticos seriam seus efeitos anticolinérgicos, anti-histaminérgicos, antagonismo dos receptores de serotonina, além da interferência da predisposição genética. $\mathrm{O}$ efeito anticolinérgico leva à boca seca, aumentando o consumo de líquidos calóricos; os efeitos anti-histaminérgicos podem levar à sedação, o que diminui a atividade e a movimentação; o antagonismo dos receptores de serotonina leva ao aumento do consumo de alimentos calóricos, fatos que colaboram para o ganho de peso. Além desses já citados, a variação genética também pode influenciar no ganho ponderal significativo após a terapia com olanzapina ou risperidona (ATTUX et al., 2009).

Nosso estudo verificou que, os medicamentos psicotrópicos utilizados pelos indivíduos eram os típicos (primeira geração), atípicos (segunda geração) e clozapina, que, embora seja um medicamento atípico, tem uma maior associação com ganho de peso quando comparado aos demais, como citado em Leitão-Azevedo et al., 2006, que associou a clozapina, além da olanzapina, com maior ganho de peso em relação aos demais antipsicóticos.

Em um estudo realizado por Zortéa et al., 2010, não houve associação entre as categorias de antipsicóticos e a classificação do IMC, ou seja, todos os pacientes em tratamento com essas drogas, independente da 
categoria, apresentaram ganho de peso, confirmando a necessidade de intervenção não farmacológica para controlar o ganho ponderal dessa população.

\section{CONSIDERAÇÕES FINAIS}

É possível afirmar, de acordo com a avaliação do estado nutricional, que o excesso de peso esteve presente em $61 \%$ dos usuários avaliados. Todas as mulheres e $59 \%$ dos homens apresentaram circunferência da cintura aumentada, indicando risco para desenvolvimento de complicações metabólicas e doenças cardiovasculares.

Faz-se necessário mapear a situação nutricional e de saúde das pessoas com transtornos mentais, promovendo atenção nutricional no SRT do município, realizada por nutricionista num contexto multiprofissional e interdisciplinar. Ressaltamos, então, a importância da ampliação do número de nutricionistas na Atenção Básica da rede municipal de saúde de

\section{AGRADECIMENTOS}

Às professoras Daniela Braga Lima e Eveline Monteiro Cordeiro de Azeredo, da
Quase metade deles (41\%) já apresentava DCNT e nenhum apresentava acompanhamento nutricional feito por nutricionista.

Sugerem-se estudos analíticos para verificar a associação entre o estado nutricional e densidade domiciliar de indivíduos usuários do SRT.

Não foi possível obter informações dos medicamentos utilizados de forma individualizada, impossibilitando a realização de análises estatísticas associando estes com o estado nutricional dos usuários, sendo essa uma limitação do estudo. Sugere-se que tais dados sejam obtidos e analisados em estudos posteriores.

Alfenas (MG), para melhoria da qualidade de vida e dos cuidados prestados a população em geral e, principalmente, a esse grupo populacional, o qual pode apresentar várias alterações em seu estado nutricional, não dispondo, ainda, da devida atenção primária a sua saúde.

Faculdade de Nutrição, UNIFAL-MG, pela subcoordenação do projeto de extensão "De perto ninguém é normal: educação em nutrição e saúde para pessoas com transtorno mental".

Ao professor Denismar Alves Nogueira, pela colaboração inicial na análise 
estatística, e à professora Luciene Alves Moreira Marques, pelo esclarecimento sobre os medicamentos antipsicóticos, ambos docentes da UNIFAL-MG.

Ao apoio de todos os usuários e profissionais do Serviço Residencial Terapêutico (SRT) de Alfenas/MG.

\section{REFERÊNCIAS BIBLIOGRÁFICAS}

1. ATTUX, C. et al. Intervenções não farmacológicas para manejo do ganho de peso em pacientes com esquizofrenia em uso de antipsicóticos. Arquivo brasileiro de endocrinologia e metabolismo, 2009.

2. BRASIL. Ministério da Saúde. DATASUS. Brasília (DF): MS; 2008. Disponível em: $<$ http://www.datasus.gov.br/cid10/V2008/We bHelp/f20_f29.htm $>$. Acesso em: 25 fev. 2013.

3. BRASIL. Ministério da Saúde. Secretaria de Atenção à Saúde, Departamento de Ações Programáticas Estratégicas. Reforma Psiquiátrica e política de Saúde Mental no Brasil. Brasília (DF): MS; 2005.

4. BRASIL. Ministério da Saúde. Orientações para a coleta e análise de dados antropométricos em serviços de saúde. Norma Técnica - SISVAN. 2008.

\section{COSTA, A. S. F. Obesidade e síndrome} metabólica na esquizofrenia. 2009. 59f. Monografia - Faculdade de Ciências da Nutrição e Alimentação, Universidade do Porto, 2009.

6. GAMA, C. S. et al. Relato do uso de clozapina em 56 pacientes atendidos pelo programa de atenção à esquizofrenia refratária da secretaria da saúde e do meio ambiente do estado do Rio Grande do Sul. Revista de Psiquiatria do Rio Grande do Sul, Rio Grande do Sul, p. 21-28, jan./abr., 2004.

7. JEKEL, J. F.; KATZ, D. L.; ELMORE, J. G. Epidemiologia, Bioestatística e Medicina Preventiva. $2^{\text {a }}$ ed. Porto Alegre: Artmed, 2005.

\section{LEITÃO-AZEVEDO, C. L. Estudo de} síndrome metabólica em pacientes com esquizofrenia tratados com antipsicóticos. 2006. 107f. Dissertação (Mestrado em Nutrição) - Faculdade de Medicina, Universidade Federal do Rio Grande do Sul, Porto Alegre, 2006.

\section{LEITÃO-AZEVEDO, C. L. et al.} Sobrepeso e obesidade em pacientes esquizofrênicos em uso de clozapina 
comparado como uso de outros antipsicóticos.

\section{Revista de Psiquiatria do Rio Grande do}

Sul, Rio Grande do Sul, p. 120-128, mai./ago., 2006.

10. LEITÃO-AZEVEDO, C. L. et al. Ganho de peso e alterações metabólicas em esquizofrenia. Revista de Psiquiatria

Clínica, v. 34, p. 184-188, 2007.

11. MARTINS, G.C.S. et al. O processo de implantação de residências terapêuticas em Volta Redonda - Rio de Janeiro. Texto

Contexto Enfermagem, Florianópolis, p. 8694, jan./mar., 2012.

\section{MELO, M.E. Os números da obesidade} no Brasil: VIGITEL 2009 e POF 2008-

2009. Disponível em:

http://www.abeso.org.br/pdf/Obesidade\%20n o\%20Brasil\%20VIGITEL\%202009\%20POF2 008_09\%20\%20II.pdf. Acesso em: 10 de abril de 2013.

13. REIS, J. S. et al. Diabetes mellitus associado com drogas antipsicóticas atípicas: relato de caso e revisão da literatura.

\section{Arquivos brasileiros de endocrinologia e} metabologia, São Paulo, v.51, n.3, abr., 2007.

14. RISSIN, A. et al. Condições de moradia como preditores de riscos nutricionais em crianças de Pernambuco, Brasil. Revista brasileira de saúde materno infantil.

Recife, v. 6, n.1, p. 59-67, jan./mar. 2006.

15. ROHMOUNEL, H. et al. Explorando o componente inflamatório da esquizofrenia. Revista de Psiquiatria Clínica, São Paulo, v. 40, n. 1, p. 28-34, 2012.

\section{SOCIEDADE BRASILEIRA DE} CARDIOLOGIA. I Diretriz brasileira de diagnóstico e tratamento da síndrome metabólica, abr., 2005.

\section{STRAKER, D. D. O. et al. Custo} benefício de triagem para a síndrome metabólica em pacientes tratados com medicamentos antipsicóticos de segunda geração. Am J Psychiatry, 2005.

18. TEIXEIRA, P. J. R; ROCHA, F. L. Associação entre síndrome metabólica e transtorno mental. Rev. Psiqui. Clin. v.34, n.5, p. 28-38, 2007.

19. VARGAS, T. S.; SANTOS, Z. E. A. Prevalência de síndrome metabólica em pacientes com esquizofrenia. Scientia Medica, Porto Alegre, v. 21, n. 1, p. 4-8, 2011.

20. ZORTÉA, K. et al. Estado nutricional de pacientes com esquizofrenia frequentadores 
do Centro de Atenção Psicossocial (CAPS) do Hospital de Clínicas de Porto Alegre. Jornal Brasileiro de Psiquiatria, Porto Alegre, v. 59, n.2, p. 126-130, 18 jun. 2010.

21. ZUGNO, A. I. et al. Energy metabolism, leptin, and biochemical parameters are altered in rats subjected to the chronic administration of olanzapine. Revista Brasileira de

Psiquiatria, v. 34, n.2, p. 168-175, jun., 2012. 